\title{
A quantitative analysis of the effect of laser transformation hardening on crack driving force in steels
}

\author{
B.Q. Yang ${ }^{\text {a,b,c,*, K. Zhang }}{ }^{\text {a }}$, G.N. Chen ${ }^{\text {a }}$, G.X. Luo ${ }^{\text {a }}$, J.H. Xiao ${ }^{\text {a }}$ \\ ${ }^{a}$ Laboratory of surface modification, Institute of mechanics, Chinese Academy of Sciences, No. 15 Beisihuanxi Road, Beijing 100080, China \\ ${ }^{\mathrm{b}}$ Department of Engineering Mechanics, Armored Force Engineering Institute, Beijing 100072, China \\ ${ }^{\mathrm{c}}$ Graduate School of the Chinese Academy of Sciences, Beijing 100080, China
}

Received 17 October 2005; accepted in revised form 21 March 2006

Available online 2 May 2006

\begin{abstract}
A mechanical model of a laser transformation hardening specimen with a crack in the middle of the hardened layer is developed to quantify the effects of the residual stress and hardness gradient on crack driving force in terms of $J$-integral. It is assumed that the crack in the middle of the hardened layer is created after laser transformation hardening. Using a Double Cantilever Beam model, the analytic solutions, which can be used to quantify the effects of the residual stress and the hardness gradient resulting from laser transformation hardening on crack driving force, are obtained. A numerical example shows the crack driving force decrease is very sensitive to the residual compressive stress increase.
\end{abstract}

(C) 2006 Elsevier B.V. All rights reserved.

Keywords: Laser transformation hardening; Crack driving force; Residual compressive stress; Hardness gradient

\section{Introduction}

The technology of laser transformation hardening (LTH) is a suitable technique for improving properties of steel surface. Authors' report [1-7] stated it could improve the fatigue properties of steels, such as the increase of the fatigue limit, fatigue strength, or the decrease of the fatigue crack growth rate. Authors' report [8] stated the laser-processed specimens could exhibit a higher resistance to crack growth in the low stress intensity factor range. It was also reported that the laser heat treatment could improve the wear properties [9-12] and the erosion properties $[13,14]$.

From the studies mentioned above, the mechanisms responsible for the prolonged lives of workpieces or specimens by LTH can be attributed to two important factors. One is the change of the mechanical property due to the changes of microstructure. Authors [15] stated that the grain size of laserhardened zone is much smaller than that of the traditional quenched high-speed steels at different quenching tempera-

\footnotetext{
* Corresponding author. Graduate School of the Chinese Academy of Sciences, Beijing 100080, China.

E-mail address: yangbq1022@tom.com (B.Q. Yang).
}

tures, and authors [16] stated a finer microstructure could be obtained by using optimum processing parameters. The other is the generation of residual compressive stress, which depends on the heating conditions and overlapping degree of the laser trace. The desirable microstructure containing fewer weak sites can exhibit a favorable combination of hardness and toughness, as stated in [14]. Hardness, the important property, can be increased greatly, and it can be measured directly or predicted by a mathematical model [17]. In general, it varies from the surface to the interior along the heat-treated track depth direction, which is termed as hardness gradient effect in this work. The results of microstructure changes, high hardness distribution and generation of high residual compressive stress by LTH can be used to interpret the fact that the technology has significantly prolonged the lives of workpieces in surface engineering. Apart from the wear and erosion experiments, it should be emphasized that most of the experiments in studies mentioned above were restricted to the fatigue property research under dynamic loads, and a rigorous and quantitative theoretical analysis of the effect after LTH treatment on crack driving force under non-dynamic loads is still lacking. The theoretical analysis is essential to predict the crack driving force of a specimen after LTH 


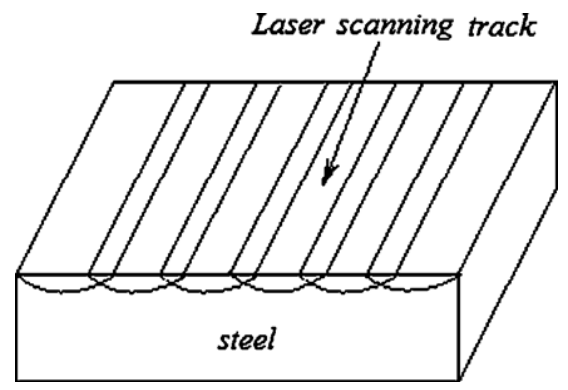

Fig. 1. A schematic illustration of the treatment way of LTH (in the form of laser scanning).

treatment, and it is also essential to the investigation of the effect after LTH treatment on the fracture toughness of a specimen. In this work, a mechanical model, based on smallscale-yielding linear elastic fracture mechanics theory, is developed that allows us to derive analytical solutions to quantify the influences of the residual stress and the hardness gradient resulting from LTH on crack driving force in terms of $J$-integral.

\section{The mechanical model}

The schematic illustration of the treatment way of LTH (in the form of laser scanning) of the steels is shown in Fig. 1. The zones between tracks are assumed to be overlapping zones. Here, the laser-scanning track is assumed to be periodic for relatively large size flat workpieces, and the interaction between the tracks is ignored for simplicity. So we can choose a representative track as our study objective of interest. In fact, a representative track is also suitable for the flat workpieces, whose surface dimension in any direction is smaller than or equal to that of the laser track. The crosssection shape of the heat treatment zone is also simplified, and a representative track is illustrated in Fig. 2, in which $h$ denotes the total depth of the heat-treated layer. As a matter of fact, laser transformation hardening of steels makes the steels change into a functional material, since the laser heattreated layer is considered to be composed of hardened zone, transition zone, heat-affected zone and base material, and each zone has different properties [18]. The transition zone consisted of a partly austenitised and eventually hardened structure and material fractions that did not transform to

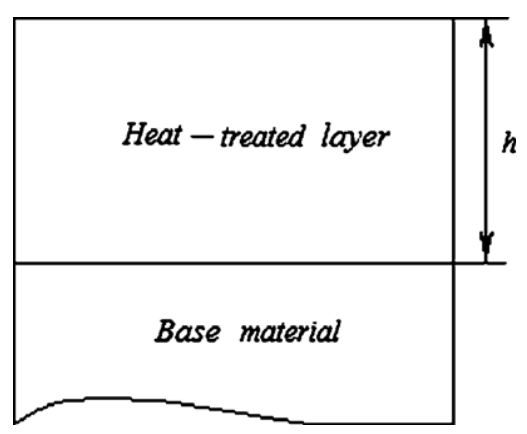

Fig. 2. A schematic illustration of the cross-section of a representative track.

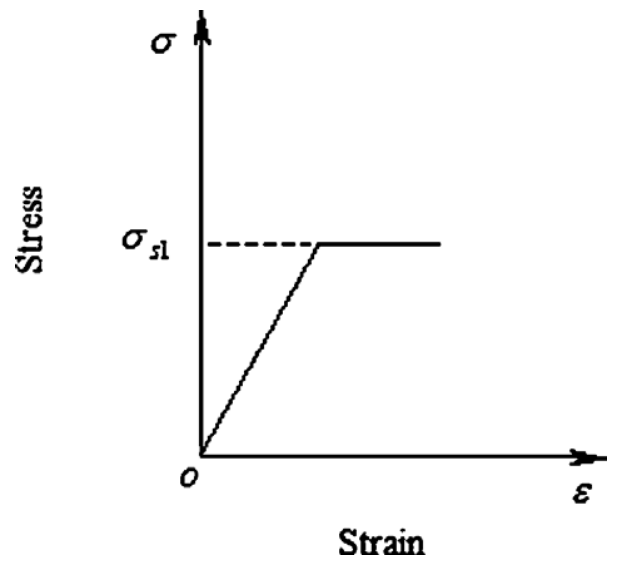

Fig. 3. The curve of the stress vs. strain of the untreated layer.

austenite during the laser irradiation, and the heat-affected zone depended on the state of the base material [18]. But in this work, the mechanical property, Young's modulus, is assumed to be invariable in the four zones since the general heat treatments have little influence on Young's modulus of metals besides LTH [19]. The stress $\sigma$ vs. strain $\varepsilon$ curve of the untreated layer and treated layer is shown in Figs. 3 and 4 , respectively. Where $\sigma_{\mathrm{s} 1}$ and $\sigma_{\mathrm{s} 2}$ are the yield strength of the untreated layer and treated layer, respectively, and $\sigma_{\mathrm{s} 2}>\sigma_{\mathrm{s} 1}$.

It should be emphasized that, for materials treated by LTH, the direct measurement of the yield strength for a particular point along the heat-treated track depth direction is very difficult to be carried out. So the law of the variable yield strength in a microzone may be hard to be obtained from a practical viewpoint. However, the measurement of the hardness is easy and quick to be performed. In addition, the microhardness could be approximated as three times of the yield strength for metal materials under the condition that the working hardening could be neglected, as stated in [20,21]. Recently, using dimensional analysis and finite element calculations,

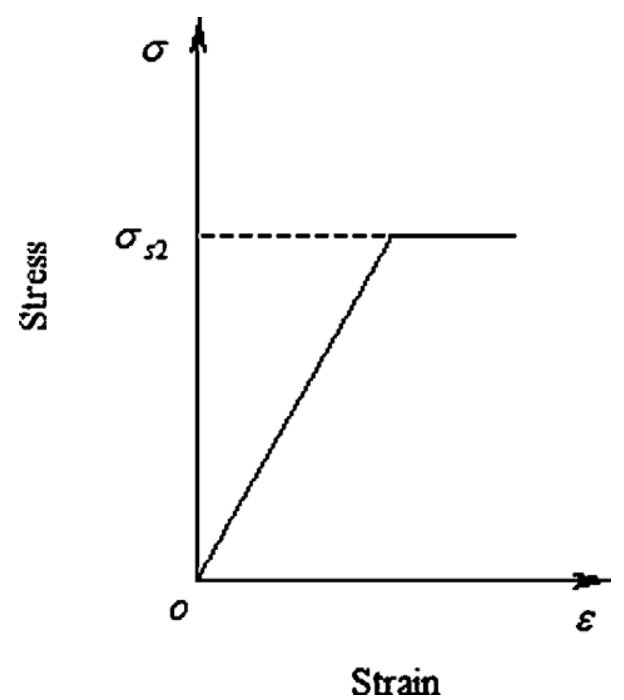

Fig. 4. The curve of the stress vs. strain of the treated layer. 
authors [22] stated the yield strength could be estimated directly from the hardness measurement in practice, and the relationship between the hardness and yield strength has been discussed in detail in $[22,23]$.

In this study, let $x$ be the abscissa denoting the heat-treated track depth direction, and the hardness in the middle of the heattreated track as a function of heat-treated track depth is assumed to obey the following law

$H=H(x)$

Hardness in the middle of the heat-treated track as a function of heat-treated track depth is illustrated in Fig. 5, in which $H_{\mathrm{m}}$ and $H_{0}$ denote the surface hardness and the hardness of the base material, respectively. One specific law of a hardness variation by hypothesis is given in Section 4 of the paper.

As shown in Fig. 6, it is assumed that there exists a crack in the middle of the hardened layer, which will propagate along the hardened track depth direction. This is a reasonable assumption since the surface and subsurface crack initiations occur independently of the applied load level, as stated in [2]. In this work, it should be emphasized the crack length, $a$, is assumed to be shorter than the hardened layer depth and much shorter than the total heat-treated layer depth (the total heat-treated layer depth is larger than four times of the crack length). Crack propagation along the hardened track depth direction in combination with the hardness variation is depicted in Fig. 7.

In this work, it should be emphasized that a pre-cracked body with a constant thickness, $B$, consisting of non-linear elastic material is assumed to have (during the loading) the same stress-strain response as an elastic-perfectly plastic material with a yield strength, as shown in Fig. 4. According to the theory of elasticity and dimension analysis, if the body with a crack were linear elastic, the potential energy, $\Pi$, would depend on the load, $F_{\mathrm{p}}$, the crack length, $a$, the Young's modulus, $E$, and the geometry of the body, $g$. For our nonlinear elastic body with yield strength, $\sigma_{\mathrm{s}}$, and residual stress,

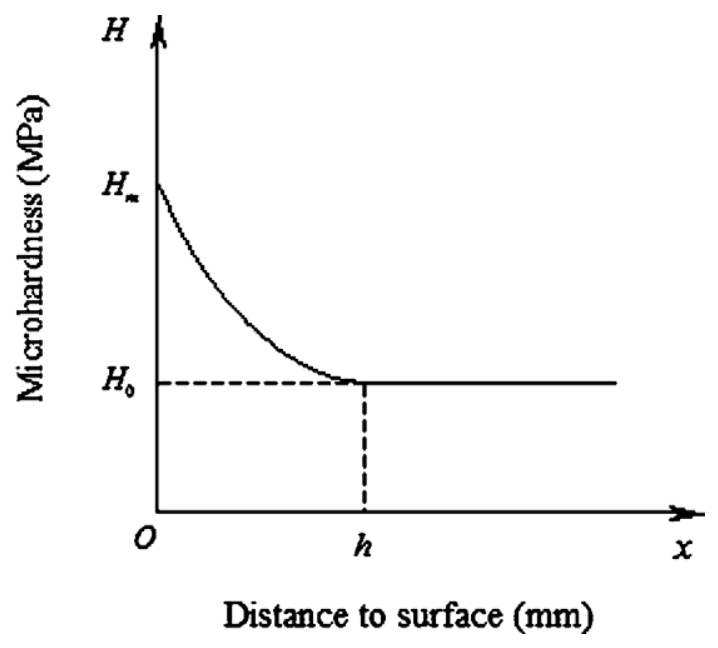

Fig. 5. Hardness as a function of the heat-treated track depth.

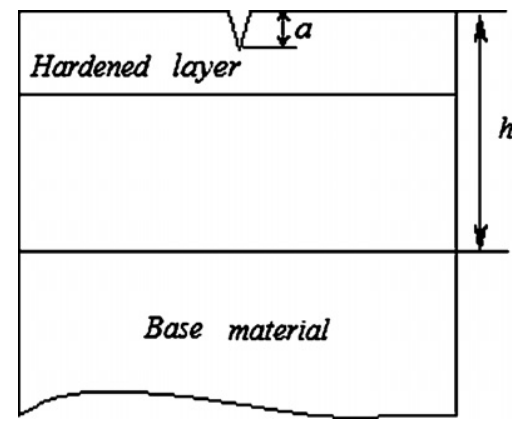

Fig. 6. A schematic illustration of a crack in the middle of the hardened layer.

$\sigma_{\mathrm{R}}$, the potential energy $\Pi$ depends additionally on $\sigma_{\mathrm{s}}$ and $\sigma_{\mathrm{R}}$, i.e.

$\Pi=\Pi\left(F_{\mathrm{p}}, a, E, g, \sigma_{\mathrm{s}}, \sigma_{\mathrm{R}}\right)$

Since the microhardness and yield strength share a same dimension and a certain relationship between them exists for a given material, we assume that the yield strength is a function of the hardness, i.e.

$\sigma_{\mathrm{s}}=\sigma_{\mathrm{s}}(H)$

Thus Eq. (2) can be written as

$\Pi=\Pi\left(F_{\mathrm{p}}, a, E, g, \sigma_{\mathrm{s}}(H), \sigma_{\mathrm{R}}\right)$

Now a thought experiment is performed:

First let the crack in the body advance by an increment, $\Delta a$, while the other parameters in Eq. (4) remain constant during the crack extension, it will deduce the well-known $J$-integral [24]

$J=-\left.\frac{1}{B} \frac{\partial \Pi}{\partial a}\right|_{\left(F_{\mathrm{p}}, E, g, \sigma_{\mathrm{s}}, \sigma_{\mathrm{R}}\right)}$

And that $J$ is a measure of the crack driving force. Then let the hardness increase by an increment, $\Delta H$, while the other parameters in Eq. (4) remain constant. Hence, if the hardness of

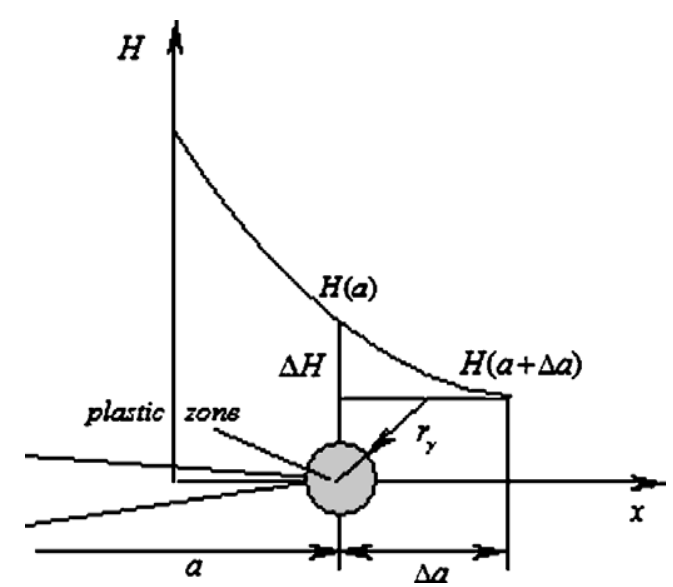

Fig. 7. A description of crack propagation in combination with hardness variation. 
the body changes as the crack propagates, an additional term of the crack driving force will appear, given by

$J_{\mathrm{H}}=-\left.\frac{1}{B} \frac{\partial \Pi}{\partial \sigma_{\mathrm{s}}}\right|_{\left(F_{\mathrm{p}}, a, E, g, \sigma_{\mathrm{R}}\right)} \frac{\mathrm{d} \sigma_{\mathrm{s}}}{\mathrm{d} H} \cdot \frac{\mathrm{d} H}{\mathrm{~d} a}$

$J_{\mathrm{H}}$ is hereafter referred to as the hardness gradient term. Thus, the total crack driving force, $J_{\text {tot }}$, is the sum of the terms in Eqs. (5) and (6)

$J_{\text {tot }}=J+J_{\mathrm{H}}$

Eq. (7) can be reduced to the case without LTH treatment. In the case of no LTH treatment on the specimen, the hardness gradient term $J_{\mathrm{H}}$ and the residual stress $\sigma_{\mathrm{R}}$ vanish, i.e. $\mathrm{d} H /$ $\mathrm{d} a=0, J_{\mathrm{H}}=0$, and $\sigma_{\mathrm{R}}=0$. The case of the specimen without LTH treatment can be dealt with by using traditional fracture mechanics method.

\section{Application of the Double Cantilever Beam model}

In order to get the specific expression of the total crack driving force, $J_{\text {tot }}$ a Double Cantilever Beam (DCB) specimen, i.e. a specimen composed of two cantilever beams, is adopted. The Double Cantilever Beam model has proved to be successful in obtaining analytical solutions for the modulus or yield strength gradient problems in inhomogeneous materials $[25,26]$. In this paper, it is extended to study the hardness gradient effect in combination with residual stress analysis, as shown in Fig. 8.

Here, the Double Cantilever Beam specimen is subjected to a pair of external loads $F_{\mathrm{p}}$, applying at the left end points. The residual stress, $\sigma_{\mathrm{R}}$, is assumed to be a pair of external loads applied to the upper and bottom boundaries of the DCB specimen, and it is compressive. Since the crack length in this paper is assumed to be shorter than the hardened layer depth and much shorter than the total heat-treated layer depth, the residual stress can be approximated as a mean value over the crack length.

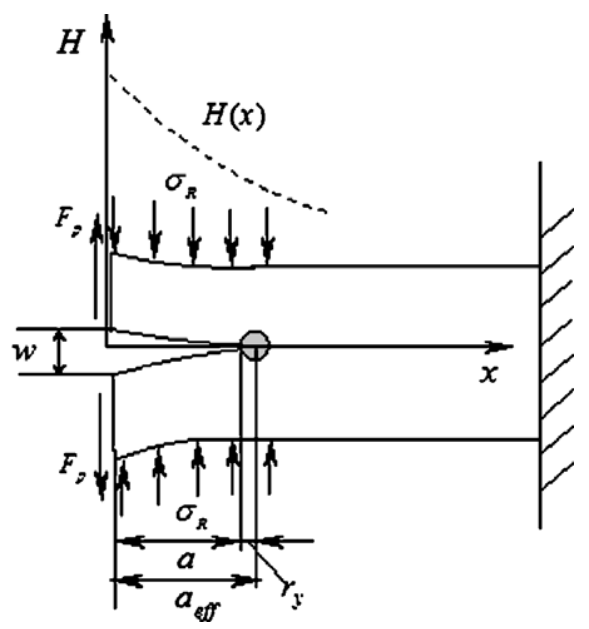

Fig. 8. A Double Cantilever Beam model.
When the crack length is equal to $a$, based on the cantilever beam theory, the vertical elastic displacement at the left end produced by a given load, $F_{\mathrm{p}}$, is

$w_{F_{\mathrm{p}}}=\frac{2 F_{\mathrm{p}} a^{3}}{3 E I}$

The vertical elastic displacement at the left end produced by the residual stress $\sigma_{\mathrm{R}}$ is

$w_{\sigma_{\mathrm{R}}}=-\frac{\sigma_{\mathrm{R}} B a^{4}}{4 E I}$

where $I$ is the moment of inertia of the cross-section of one beam and $B$ is the constant thickness of the body. Then the total vertical elastic displacement at the left end in the direction of the load $F_{\mathrm{p}}$ is the sum of the terms in Eqs. (8) and (9)

$w_{\mathrm{e}}=\frac{2 F_{\mathrm{p}} a^{3}}{3 E I}-\frac{\sigma_{\mathrm{R}} B a^{4}}{4 E I}$

For the small-scale yielding conditions, Irwin's model of a circular plastic zone at the crack tip is adopted, and the radius is given by [27]

$r_{\mathrm{y}}=\beta \frac{G E}{\sigma_{\mathrm{s}}^{2}}$

where $\sigma_{\mathrm{s}}$ is the yield strength at the center of the plastic zone. Note that at a given load, $\sigma_{\mathrm{s}}$ determines the radius $r_{\mathrm{y}}$ of the plastic zone, and from Eq. (11), we can see the radius of the circular plastic zone is inversely proportional to squared yield strength. Throughout this paper, $r_{\mathrm{y}}$ is assumed to be small against the crack length $a$. For plane strain problems, $\beta$ is a constant and equals to $1 /(6 \pi)$ [27]. $G$ is the strain energy release rate, which is defined as the strain energy release per unit crack area. For the DCB specimen, $G$ can be calculated and the result is

$G=\frac{F_{\mathrm{p}}^{2} a^{2}}{B E I}-\frac{F_{\mathrm{p}} \sigma_{\mathrm{R}} a^{3}}{2 E I}$

The specific relationship between microhardness and yield strength can be written as [20-23]

$\sigma_{\mathrm{s}}=k H$

where $k$ is a dimensionless coefficient. For a given material, the coefficient $k$ depends on the properties of the material and can be determined by experiment [20] or finite element calculations $[22,23]$. In the case of the elastic-perfectly plastic metals, for most metals for which $0<\sigma_{\mathrm{s}} / E<0.01, k$ is between $1 / 2.6$ and 1/ 2.5 , as stated in [23]. For a variety of materials, $k$ can range from $1 / 2.8$ to $1 / 2.7$, but for most metals for which $0<\sigma_{\mathrm{s}} / E<0.01, k$ approximates $1 / 2.8$, as stated in [22]. But authors [20,21] stated that the coefficient $k$ could be approximated as $1 / 3$ under the condition that the working hardening could be neglected. After using dimensional analysis and the results presented in $[22,23]$, the hardness variation rate can represent the variation rate of the 
yield strength because the laser heat-treated layer and the base material share a same Young's modulus and the non-linear elastic material is assumed to be elastic-perfectly plastic in this work.

Substituting Eq. (13) into Eq. (11) yields

$r_{\mathrm{y}}=\beta \frac{G E}{k^{2} H^{2}}$

Including the displacement contributed by perfect plasticity, a good estimate of the total displacement at the left end in the direction of the load $F_{\mathrm{p}}$ can be obtained by replacing in Eq. (10) the crack length by the effective crack length, $a_{\mathrm{eff}}=a+r_{\mathrm{y}}$, i.e.

$w=\frac{2 F_{\mathrm{p}} a^{3}}{3 E I}\left(1+\frac{r_{\mathrm{y}}}{a}\right)^{3}-\frac{\sigma_{\mathrm{R}} B a^{4}}{4 E I}\left(1+\frac{r_{\mathrm{y}}}{a}\right)^{4}$

Thus, the total displacement $w$ at the left end in the direction of the load $F_{\mathrm{p}}$, which is composed of the displacements contributed by the load $F_{\mathrm{p}}$, the residual stress $\sigma_{\mathrm{R}}$ and the perfect plasticity, is obtained. As was pointed out by Rice [28], the potential energy for a load-controlled test, $\left.\Pi\right|_{F_{\mathrm{p}}}$ can be calculated from

$\left.\Pi\right|_{F_{\mathrm{p}}}=-\int_{0}^{F_{\mathrm{p}}} w \mathrm{~d} F_{\mathrm{p}}$

Using Eqs. (12) and (14), the specific potential energy can be calculated by substituting Eq. (15) into Eq. (16), and the result is

$\left.\Pi\right|_{F_{\mathrm{p}}}=\sum_{i=1}^{9} C_{i} F_{\mathrm{p}}^{i}$

where $C_{i}(i=1,2,3, \ldots, 9)$ are given in Appendix A.

Eq. (17), together with Eqs. (5), (6) and (7), leads to the desired analytical solutions

$J=\sum_{i=2}^{11} A_{i} a^{i}$

$J_{\mathrm{H}}=\sum_{i=1}^{4} \lambda_{-(2 i+1)} H^{-(2 i+1)} \frac{\mathrm{d} H}{\mathrm{~d} x}$

$J_{\mathrm{tot}}=J+J_{\mathrm{H}}=\sum_{i=2}^{11} A_{i} a^{i}+\sum_{i=1}^{4} \lambda_{-(2 i+1)} H^{-(2 i+1)} \frac{\mathrm{d} H}{\mathrm{~d} x}$

where $A_{i}(i=2,3,4, \ldots, 11)$ are given in Appendix B, and $\lambda_{-(2 i+1)}$ $(i=1,2,3,4)$ are given in Appendix C.

Eq. (18) is the term of the crack driving force that includes the residual stress, and Eq. (19) is the other term of the crack driving force that includes the hardness gradient. Thus, the total

Table 1

Some parameters used for the calculations

\begin{tabular}{llllll}
\hline$E(\mathrm{GPa})$ & $h(\mathrm{~mm})$ & $a(\mathrm{~mm})$ & $B(\mathrm{~mm})$ & $I\left(\mathrm{~mm}^{4}\right)$ & $\beta$ \\
\hline 210 & 0.6 & 0.1 & 0.02 & $1.33 \times 10^{-8}$ & $1 /(6 \pi)$ \\
\hline
\end{tabular}

Table 2

The crack driving force $J_{\text {tot }}^{R}\left(\mathrm{~J} / \mathrm{m}^{2}\right)$ vs. the residual compressive stress $\sigma_{\mathrm{R}}(\mathrm{MPa})$

\begin{tabular}{lllllllll}
\hline$J_{\text {tot }}^{R}$ & 19523 & 16149 & 13011 & 10096 & 7394.2 & 4894.6 & 2587.2 & 462.18 \\
$\sigma_{\mathrm{R}}$ & 200 & 210 & 220 & 230 & 240 & 250 & 260 & 270 \\
\hline
\end{tabular}

crack driving force expressed in Eq. (20), which is the sum of the two terms, is obtained.

\section{A numerical example}

A numerical example shall assess the influences of the residual compressive stress and the hardness gradient on crack driving force. The hardness gradient in the middle of the heattreated track depth direction $x$ is assumed to follow an exponential function

$H(x)=D_{0} \exp (\delta x)$

where $D_{0}$ and $\delta$ are the constants to be determined. From Fig. 5, it can be seen when $x=0, H=H_{\mathrm{m}}=D_{0}$, and when $x=h$, $H=H_{0}=D_{0} \exp (\delta h)$. Once the hardness values at the two points are known, the constants $D_{0}$ and $\delta$ can be determined.

For a particular case, one of the hardness distribution characteristics in the middle of the heat-treated track along the heat-treated track depth direction is taken from [14], in which when $x=0, H$ approximately equals to $8 \times 10^{9} \mathrm{~Pa}$, and when $x=h=6 \times 10^{-4} \mathrm{~m}, H$ approximately equals to $3 \times 10^{9} \mathrm{~Pa}$. Using Eq. (21), the constants $D_{0}$ and $\delta$ can be determined and the corresponding hardness variation law is given as

$H(x)=8.0 \times 10^{9} \exp (-1634.7 x)$

Some parameters used for the calculations of $J, J_{\mathrm{H}}$ and $J_{\text {tot }}$ are given in Table 1. All the parameters in Table 1 are invariant throughout this calculation. The external load $F_{\mathrm{p}}$ is also assumed to remain constant and equal to $0.5 \mathrm{~N}$ throughout this calculation. The residual compressive stress

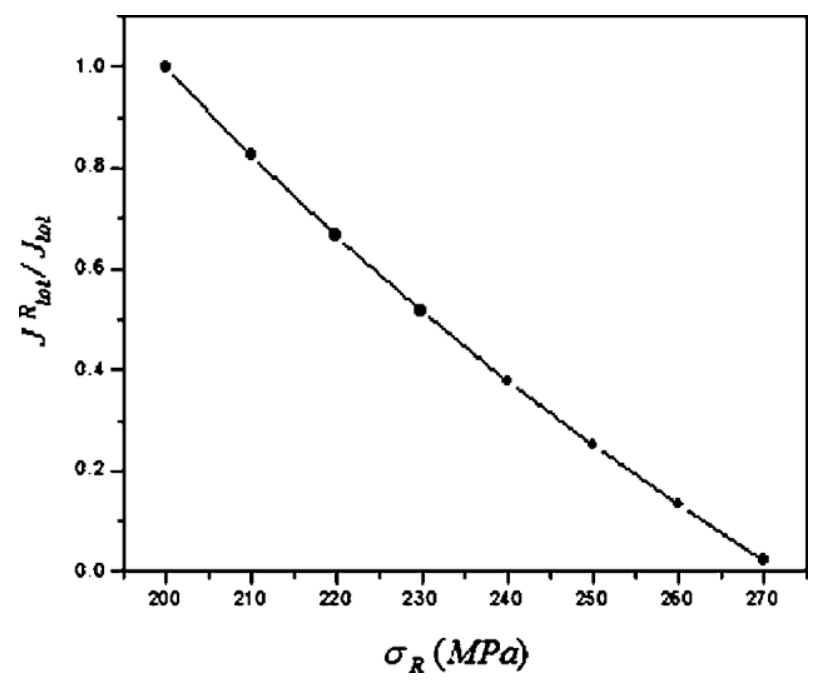

Fig. 9. Plot of the ratio $J_{\text {tot }}^{R} / J_{\text {tot }}$ vs. the residual compressive stress $\sigma_{\mathrm{R}}$. 
Table 3

The crack driving force $J_{\text {tot }}^{k^{\prime}}\left(\mathrm{J} / \mathrm{m}^{2}\right)$ vs. the coefficient $k^{\prime}\left(k^{\prime}=1 / k\right)$

\begin{tabular}{llllllll}
\hline$J_{\text {tot }}^{k^{\prime}}$ & 13,806 & 15,047 & 16,423 & 17,922 & 19,523 & 21,192 & 23,714 \\
$k^{\prime}$ & 1.7 & 1.9 & 2.1 & 2.3 & 2.5 & 2.7 & 3 \\
\hline
\end{tabular}

on the surface and in the surface layer down to a certain depth can range between 200 and $500 \mathrm{MPa}$, as stated in [29]. For the first calculation, the value of the residual compressive stress is assumed to equal to $200 \mathrm{MPa}$, and $k$ is assumed to equal to $1 / 2.5$ [23]. Given these conditions, $J_{\text {tot }}$ can be calculated and it equals to $1.9523 \times 10^{4}\left(\mathrm{~J} / \mathrm{m}^{2}\right)$. It should be emphasized this value is also a normalized factor for the following analysis.

In this work, we investigate the influence of the variation of the residual compressive stress on the crack driving force. Let $J_{\text {tot }}^{\mathrm{R}}$ denote the crack driving force of different residual compressive stresses, and the crack driving force $J_{\text {tot }}^{\mathrm{R}}$ vs. the residual compressive stress $\sigma_{\mathrm{R}}$ is shown in Table 2. $J_{\text {tot }}^{\mathrm{R}}$ is normalized with respect to $J_{\text {tot }}$ mentioned above, and the plot of the ratio $J_{\text {tot }}^{\mathrm{R}} / J_{\text {tot }}$ vs. the residual compressive stress $\sigma_{\mathrm{R}}$ is shown in Fig. 9.

For convenience, we define another dimensionless coefficient $k^{\prime}$, which is equal to $1 / k$. In this work, based on the results [20-23], the coefficient $k^{\prime}$ linking the hardness and yield strength is assumed to range from 1.7 to 3.0. Let $J_{\text {tot }}^{k^{\prime}}$ denote the crack driving force of different coefficients, and the crack driving force $J_{\text {tot }}^{k^{\prime}}$ vs. the coefficient $k^{\prime}$ is show in Table $3 . J_{\text {tot }}^{k^{\prime}}$ is also normalized with respect to $J_{\text {tot }}$, and the plot of the variation of $k^{\prime}$ on the ratio $J_{\text {tot }}^{k^{\prime}} / J_{\text {tot }}$ is shown in Fig. 10.

\section{Results and discussions}

From Table 2 and Fig. 9, it can be seen that the crack driving force decreases with the increase of the residual compressive stress. From Table 2, it can also be calculated easily as the residual compressive stress increases per $10 \mathrm{MPa}$, the least decrease rate of the crack driving force lies between 200 and $210 \mathrm{MPa}$ and the decrease rate is $17.28 \%$, while the largest decrease rate lies between 260 and $270 \mathrm{MPa}$ and it can reach $82.14 \%$. So we can draw a conclusion that the crack driving force is very sensitive to the residual compressive stress, and the crack driving force decreases significantly with the increase of residual compressive stress. On the contrary, if the residual stress is tensile, the crack driving force will increase significantly with the increase of residual tensile stress, and it will adversely facilitate the crack propagation. This case can be detrimental to the workpieces.

From Table 3 and Fig. 10, we can see that the crack driving force increases as $k^{\prime}$ increases. This case can be explained in the following way. Apart from the case that the variation of $k^{\prime}$ can represent a variety of materials, on the other hand, the increase of $k^{\prime}$ from 1.7 to 3 also implies the decrease of the yield strength from another standpoint, because the hardness is invariant for a given crack length during this calculation. So, it is reasonable that the increase of $k^{\prime}$ (or the decrease of the yield strength) leads to the increase of the crack driving force.
From the numerical results, the higher residual compressive stress and higher strength can decrease much more crack driving force, and the crack driving force tends to be much more sensitive to the residual compressive stress. On the other hand, the decrease of the crack driving force means the increase of resistance to crack growth, and this situation can improve the fracture behavior. Besides the results presented in this work, the high residual compressive stress and high strength resulting from LTH treatment also play an important role in improving fatigue behavior [1-8], such as the decrease of the fatigue crack growth rate or the increase of resistance to crack growth $[2,5,8]$. Thus, the residual compressive stress and high strength from LTH treatment have a significant effect on fracture behavior in fatigue or non-fatigue cases. So from the numerical results mentioned above, we can conclude that the mechanical model presented in this paper is reasonable, and that the results can be used to quantify the LTH treatment effect on crack driving force. In addition, if the variation law and magnitude of the residual stress can be determined, the procedure presented in this paper can be used to study the LTH treatment effect on fracture toughness.

In order to obtain accurate results throughout the calculation, it should be noticed that the geometric parameters of the specimen must satisfy the beam model, and the radius $r_{\mathrm{y}}$ of circular plastic zone at the crack tip must satisfy the condition that they are much smaller than the crack length $a$ and specimen dimensions. At the same time, the magnitude of the external load $F_{\mathrm{p}}$, which is a constant for this calculation, must also be properly taken in that the total displacement $w$ and the elastic strain energy release rate $G$ must be positive, otherwise it would be the crack closure/contact problem.

The results obtained from the mechanical model are based on some assumptions. The crack is assumed to be in the middle of the hardened layer, and its length is assumed to be shorter than the hardened layer depth and much shorter than the total heat-treated layer depth. So the residual compressive stress can be approximated as a mean value over the crack length. The cases that the crack tip is in the hardened layer,

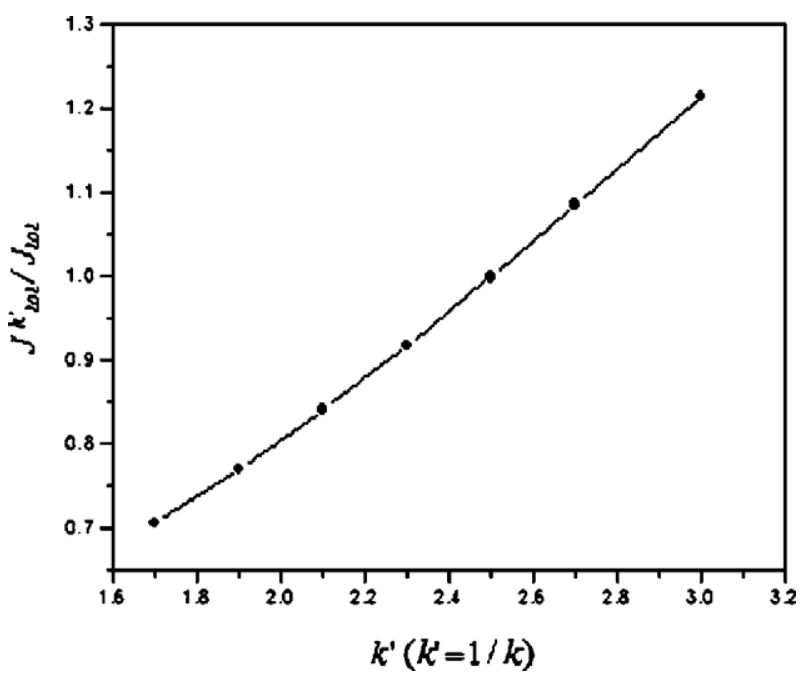

Fig. 10. Plot of the ratio $J_{\text {tot }}^{k^{\prime}} / J_{\text {tot }}$ vs. the coefficient $k^{\prime}\left(k^{\prime}=1 / k\right)$. 
transitional layer, heat-affected layer or base material are different, and they must be dealt with separately. For example, if the crack tip is in transitional layer or heataffected layer, we must consider the residual stress gradient effect, i.e. the residual stress as a function of the depth below surface, or the variation of the residual stress from compressive to tensile [29-31]. The hardness in the middle of the heat-treated track as a function of heat-treated track depth is assumed to satisfy an exponentially decaying law. One can also take other formulas to approximately express the law of the hardness variation along the heat-treated track depth direction for a specific or practical case.

The results presented in this work are not only limited to the case of LTH treatment, they can also be extended to other forms of high-energy surface treatments, such as electron surface treatment, ion surface treatment, and so on. The differences are that the magnitude and the distribution laws of the hardness and residual stress may be different for corresponding specific cases. Once the specific laws and magnitude of the residual stress and hardness are given, the procedure presented in this paper can also be adopted without any difficulty.

\section{Conclusions}

The analytical solutions presented in this paper can quantify the effects of the residual stress and hardness gradient after
LTH treatment on crack driving force in terms of $J$-integral. A numerical example shows the crack driving force is very sensitive to the residual stress.

These analytical solutions in this paper, can not only be used as a means to predict the crack driving force, but also serve as a baseline for further experimental measurement of fracture toughness, accounting for the LTH treatment effect.

The results presented in this paper are suitable for the case that the crack lies in the middle of the hardened layer, and its length is assumed to be shorter than the hardened layer depth and much shorter than the total heat-treated layer depth. The results presented in this paper are not suitable for the cases that the crack tip is in the transitional layer, heat-affected layer or base material. These cases are different, and they must be dealt with separately.

In the end it deserves to point out that, the results presented in this work can also be applied to other forms of high-energy surface treatments.

\section{Acknowledgements}

The financial support provided by the National Natural Science Foundation of China (Grant No. 50471087, 50531060 ) is greatly acknowledged. We also want to express our deep gratitude to reviewers for their constructive suggestions.

\section{Appendix A}

$$
\begin{aligned}
& \left.\Pi\right|_{F_{\mathrm{p}}}=\sum_{i=1}^{9} C_{i} F_{\mathrm{p}}^{i}=C_{1} F_{\mathrm{p}}+C_{2} F_{\mathrm{p}}^{2}+C_{3} F_{\mathrm{p}}^{3}+C_{4} F_{\mathrm{p}}^{4}+C_{5} F_{\mathrm{p}}^{5}+C_{6} F_{\mathrm{p}}^{6}+C_{7} F_{\mathrm{p}}^{7}+C_{8} F_{\mathrm{p}}^{8}+C_{9} F_{\mathrm{p}}^{9} \\
& C_{1}=\frac{\sigma_{\mathrm{R}} B a^{4}}{4 E I}, \\
& C_{2}=-\frac{a^{3}}{3 E I}-\frac{\beta B \sigma_{\mathrm{R}}^{2} a^{6}}{4 E I^{2}(k H)^{2}}, \\
& C_{3}=\frac{\beta \sigma_{\mathrm{R}} a^{5}}{3 E I^{2}(k H)^{2}}+\frac{\sigma_{\mathrm{R}} B a^{4}}{12 E I}\left(\frac{4 \beta a}{B I(k H)^{2}}+\frac{3 \beta^{2} \sigma_{\mathrm{R}}^{2} a^{4}}{2 I^{2}(k H)^{4}}\right), \\
& C_{4}=-\frac{a^{3}}{6 E I}\left(\frac{3 \beta a}{B I(k H)^{2}}+\frac{3 \beta^{2} \sigma_{\mathrm{R}}^{2} a^{4}}{4 I^{2}(k H)^{4}}\right)-\frac{\sigma_{\mathrm{R}} B a^{4}}{16 E I}\left[\frac{2 \beta^{2} \sigma_{\mathrm{R}} a^{3}}{B I^{2}(k H)^{4}}+\frac{2 \beta \sigma_{\mathrm{R}} a^{2}}{I(k H)^{2}}\left(\frac{2 \beta a}{B I(k H)^{2}}+\frac{\beta^{2} \sigma_{\mathrm{R}}^{2} a^{4}}{4 I^{2}(k H)^{4}}\right)\right], \\
& C_{5}=\frac{2 a^{3}}{15 E I}\left[\frac{2 \beta^{2} \sigma_{\mathrm{R}} a^{3}}{B I^{2}(k H)^{4}}+\frac{\beta \sigma_{\mathrm{R}} a^{2}}{2 I(k H)^{2}}\left(\frac{2 \beta a}{B I(k H)^{2}}+\frac{\beta^{2} \sigma_{\mathrm{R}}^{2} a^{4}}{4 I^{2}(k H)^{4}}\right)\right]+\frac{\sigma_{\mathrm{R}} B a^{4}}{20 E I}\left[\frac{2 \beta^{2} a^{2}}{B^{2} I^{2}(k H)^{4}}+\frac{2 \beta^{3} \sigma_{\mathrm{R}}^{2} a^{5}}{B I^{3}(k H)^{6}}+\left(\frac{2 \beta a}{B I(k H)^{2}}+\frac{\beta^{2} \sigma_{\mathrm{R}}^{2} a^{4}}{4 I^{2}(k H)^{4}}\right)^{2}\right], \\
& C_{6}=\frac{a^{3}}{9 E I}\left[-\frac{\beta^{2} a^{2}}{B^{2} I^{2}(k H)^{4}}-\frac{\beta^{3} \sigma_{\mathrm{R}}^{2} a^{5}}{2 B I^{3}(k H)^{6}}-\frac{\beta a}{B I(k H)^{2}}\left(\frac{2 \beta a}{B I(k H)^{2}}+\frac{\beta^{2} \sigma_{\mathrm{R}}^{2} a^{4}}{4 I^{2}(k H)^{4}}\right)\right]-\frac{\sigma_{\mathrm{R}} B a^{4}}{24 E I}\left[\frac{2 \beta^{3} \sigma_{\mathrm{R}} a^{4}}{B^{2} I^{3}(k H)^{6}}+\frac{\beta^{2} \sigma_{\mathrm{R}} a^{3}}{B I^{2}(k H)^{4}}\left(\frac{4 \beta a}{B I(k H)^{2}}+\frac{\beta^{2} \sigma_{\mathrm{R}}^{2} a^{4}}{2 I^{2}(k H)^{4}}\right)\right], \\
& C_{7}=\frac{\beta^{3} \sigma_{\mathrm{R}} a^{7}}{7 E B^{2} I^{4}(k H)^{6}}+\frac{\sigma_{\mathrm{R}} B a^{4}}{28 E I}\left[\left(\frac{4 \beta a}{B I(k H)^{2}}+\frac{\beta^{2} \sigma_{\mathrm{R}}^{2} a^{4}}{2 I^{2}(k H)^{4}}\right) \frac{\beta^{2} a^{2}}{B^{2} I^{2}(k H)^{4}}+\frac{\beta^{4} \sigma_{\mathrm{R}}^{2} a^{6}}{B^{2} I^{4}(k H)^{8}}\right], \\
& C_{8}=-\frac{\beta^{3} a^{6}}{12 E B^{3} I^{4}(k H)^{6}}-\frac{\sigma_{\mathrm{R}}^{2} \beta^{4} a^{9}}{16 E B^{2} I^{5}(k H)^{8}}, \\
& C_{9}=\frac{\sigma_{\mathrm{R}} \beta^{4} a^{8}}{36 E B^{3} I^{5}(k H)^{8} .}
\end{aligned}
$$




\section{Appendix B}

$$
\begin{aligned}
& J=\sum_{i=2}^{11} A_{i} a^{i}=A_{2} a^{2}+A_{3} a^{3}+A_{4} a^{4}+A_{5} a^{5}+A_{6} a^{6}+A_{7} a^{7}+A_{8} a^{8}+A_{9} a^{9}+A_{10} a^{10}+A_{11} a^{11} . \\
& A_{2}=\frac{F_{\mathrm{p}}^{2}}{E B I} \text {, } \\
& A_{3}=-\frac{\sigma_{\mathrm{R}} F_{\mathrm{p}}}{E I}+\frac{2 \beta F_{\mathrm{p}}^{4}}{E B^{2} I^{2}(k H)^{2}}, \\
& A_{4}=\frac{5 \beta^{2} F_{\mathrm{p}}^{6}}{3 E B^{3} I^{3}(k H)^{4}}-\frac{10 \sigma_{\mathrm{R}} \beta F_{\mathrm{p}}^{3}}{3 E B I^{2}(k H)^{2}}, \\
& A_{5}=\frac{3 \sigma_{\mathrm{R}}^{2} \beta F_{\mathrm{p}}^{2}}{2 E I^{2}(k H)^{2}}-\frac{21 \sigma_{\mathrm{R}} \beta^{2} F_{\mathrm{p}}^{5}}{5 E B^{2} I^{3}(k H)^{4}}+\frac{\beta^{3} F_{\mathrm{p}}^{8}}{2 E B^{4} I^{4}(k H)^{6}}, \\
& A_{6}=\frac{7 \sigma_{\mathrm{R}}^{2} \beta^{2} F_{\mathrm{p}}^{4}}{2 E B I^{3}(k H)^{4}}-\frac{2 \sigma_{\mathrm{R}} \beta^{3} F_{\mathrm{p}}^{7}}{E B^{3} I^{4}(k H)^{6}} \\
& A_{7}=-\frac{\sigma_{\mathrm{R}}^{3} \beta^{2} F_{\mathrm{p}}^{3}}{E I^{3}(k H)^{4}}+\frac{8 \sigma_{\mathrm{R}}^{2} \beta^{3} F_{\mathrm{p}}^{6}}{3 E B^{2} I^{4}(k H)^{6}}-\frac{2 \sigma_{\mathrm{R}} \beta^{4} F_{\mathrm{p}}^{9}}{9 E B^{4} I^{5}(k H)^{8}}, \\
& A_{8}=-\frac{3 \sigma_{\mathrm{R}}^{3} \beta^{3} F_{\mathrm{p}}^{5}}{2 E B I^{4}(k H)^{6}}+\frac{9 \sigma_{\mathrm{R}}^{2} \beta^{4} F_{\mathrm{p}}^{8}}{16 E B^{3} I^{5}(k H)^{8}}, \\
& A_{9}=\frac{5 \sigma_{\mathrm{R}}^{4} \beta^{3} F_{\mathrm{p}}^{4}}{16 E I^{4}(k H)^{6}}-\frac{15 \sigma_{\mathrm{R}}^{3} \beta^{4} F_{\mathrm{p}}^{7}}{28 E B^{2} I^{5}(k H)^{8}}, \\
& A_{10}=\frac{11 \sigma_{\mathrm{R}}^{4} \beta^{4} F_{\mathrm{p}}^{6}}{48 B E I^{5}(k H)^{8}}, \\
& A_{11}=-\frac{3 \sigma_{\mathrm{R}}^{5} \beta^{4} F_{\mathrm{p}}^{5}}{80 E I^{5}(k H)^{8}} \text {. }
\end{aligned}
$$

\section{Appendix C}

$$
\begin{aligned}
& J_{\mathrm{H}}=\sum_{i=1}^{4} \lambda_{-(2 i+1)} H^{-(2 i+1)} \frac{\mathrm{d} H}{\mathrm{~d} x}=\left(\lambda_{-3} H^{-3}+\lambda_{-5} H^{-5}+\lambda_{-7} H^{-7}+\lambda_{-9} H^{-9}\right) \frac{\mathrm{d} H}{\mathrm{~d} x} . \\
& \lambda_{-3}=-\frac{\beta a^{4} F_{\mathrm{p}}^{4}}{E B^{2} I^{2} k^{2}}+\frac{4 \sigma_{\mathrm{R}} \beta a^{5} F_{\mathrm{p}}^{3}}{3 E B I^{2} k^{2}}-\frac{\sigma_{\mathrm{R}}^{2} a^{6} \beta F_{\mathrm{p}}^{2}}{2 E I^{2} k^{2}}, \\
& \lambda_{-5}=\frac{14 \sigma_{\mathrm{R}} \beta^{2} a^{6} F_{\mathrm{p}}^{5}}{5 E B^{2} I^{3} k^{4}}-\frac{4 \beta^{2} a^{5} F_{\mathrm{p}}^{6}}{3 E B^{3} I^{3} k^{4}}-\frac{2 \sigma_{\mathrm{R}}^{2} a^{7} \beta^{2} F_{\mathrm{p}}^{4}}{E B I^{3} k^{4}}+\frac{\sigma_{\mathrm{R}}^{3} a^{8} \beta^{2} F_{\mathrm{p}}^{3}}{2 E I^{3} k^{4}}, \\
& \lambda_{-7}=-\frac{\beta^{3} a^{6} F_{\mathrm{p}}^{8}}{2 E B^{4} I^{4} k^{6}}-\frac{2 \sigma_{\mathrm{R}}^{2} \beta^{3} a^{8} F_{\mathrm{p}}^{6}}{E B^{2} I^{4} k^{6}}-\frac{3 \sigma_{\mathrm{R}}^{4} a^{10} \beta^{3} F_{\mathrm{p}}^{4}}{16 E I^{4} k^{6}}+\frac{12 \sigma_{\mathrm{R}} a^{7} \beta^{3} F_{\mathrm{p}}^{7}}{7 E B^{3} I^{4} k^{6}}+\frac{\sigma_{\mathrm{R}}^{3} a^{9} \beta^{3} F_{\mathrm{p}}^{5}}{E B I^{4} k^{6}}, \\
& \lambda_{-9}=\frac{2 \sigma_{\mathrm{R}} \beta^{4} a^{8} F_{\mathrm{p}}^{9}}{9 E B^{4} I^{5} k^{8}}-\frac{\sigma_{\mathrm{R}}^{2} \beta^{4} a^{9} F_{\mathrm{p}}^{8}}{2 E B^{3} I^{5} k^{8}}+\frac{3 \sigma_{\mathrm{R}}^{3} a^{10} \beta^{4} F_{\mathrm{p}}^{7}}{7 E B^{2} I^{5} k^{8}}+\frac{\sigma_{\mathrm{R}}^{5} a^{12} \beta^{4} F_{\mathrm{p}}^{5}}{40 E I^{5} k^{8}}-\frac{\sigma_{\mathrm{R}}^{4} a^{11} \beta^{4} F_{\mathrm{p}}^{6}}{6 E B I^{5} k^{8}} .
\end{aligned}
$$

\section{References}

[1] M. Heitkemper, C. Bohne, A. Pyzalla, A. Fischer, Int. J. Fatigue 25 (2003) 101.

[2] Pedro De la Cruz, Magnus Odén, Torsten Ericsson, Int. J. Fatigue 20 (1998) 389.

[3] H. Yijun, S. Deheng, Y. Hongying, Second International Conference on Optoelectronic Science and Engineering, Beijing, August 15-18 1994, p. 600 .

[4] P. Merrien, H.P. Lieurade, M. Theobalt, G. Baudry, T. Puig, F. Leroy, Surf. Eng. 8 (1) (1992) 61.
[5] J.L. Doong, T.J. Chen, Y.H. Tan, Eng. Fract. Mech. 33 (3) (1989) 483.

[6] A. Kato, J. Eng. Mater. Technol. 107 (1985) 195.

[7] H.B. Singh, S.M. Copley, M. Bass, Metall. Trans. 12A (1981) 138.

[8] L.W. Tsay, M.C. Young, C. Chen, Corros. Sci. 45 (2003) 1985.

[9] D.I. Pantelis, E. Bouyiouri, N. Kouloumbi, P. Vassiliou, A. Koutsomichalis, Surf. Coat. Technol. 298 (2002) 125.

[10] T.M. Shao, M. Hua, T.H. Yuen, Wear 255 (2003) 444.

[11] Z.D. Dai, S.C. Pan, M. Wang, S.R. Yang, X.S. Zhang, Q.J. Xue, Wear 213 (1997) 135.

[12] M. Kulka, A. Pertek, Appl. Surf. Sci. 236 (2004) 98.

[13] P.H. Chong, Z. Liu, P. Skeldon, G.E. Thompson, Appl. Surf. Sci. 208-209 (2003) 399. 
[14] K.H. Lo, F.T. Cheng, H.C. Man, Surf. Coat. Technol. 173 (2003) 96.

[15] G.Q. Shi, P.D. Ding, J.L. Liu, H.J. Yin, J. Wang, Acta Metall. Mater. 43 (1995) 217.

[16] Q.B. Liu, H. Liu, J. Mater. Process. Technol. 88 (1999) 77.

[17] Y.H. Guan, T.L. Chen, H.G. Wang, J.T. Zhang, J. Mater. Process. Technol. 63 (1997) 614.

[18] K. Obergfell, V. Schulze, O. Vöhringer, Mater. Sci. Eng., A Struct. Mater.: Prop. Microstruct. Process. 355 (2003) 348.

[19] M.A. Meyers, K.K. Chawla, Mechanical Metallurgy, Prentice Hall, New Jersey, 1984.

[20] D. Tabor, The Hardness of Metals, Oxford University Press, London, 1951.

[21] M.F. Ashby, D.R.H. Jones, Engineering Materials, Oxford Pergamon Press, London, 1980, p. 105.

[22] Y.T. Cheng, C.M. Cheng, J. Appl. Phys. 84 (1998) 1284.
[23] Y.T. Cheng, C.M. Cheng, Int. J. Solids Struct. 36 (1999) 1231.

[24] J.R. Rice, in: H. Liebowitz (Ed.), Fracture: An Advanced Treatise, Academic Press, New York, 1968, p. 191.

[25] T. Honein, G. Herrmann, J. Mech. Phys. Solids 45 (1997) 789.

[26] O. Kolednik, Int. J. Solids Struct. 37 (2000) 781.

[27] G.R. Irwin, Proceedings of the 7th Sagamore Ordnance Materials Research Conference, New York, 1960, p. IV-63.

[28] J.R. Rice, J. Appl. Mech. 35 (1968) 379.

[29] J. Grum, P. Žerovnik, Heat Treat. 25 (1993) 32.

[30] J. Grum, in: G.E. Totten, K. Funatani, L. Xie (Eds.), Handbook of Metallurgical Process Design, Marcel Dekker, New York, 2004, p. 641.

[31] J. Grum, R. Sturm, P. Žerovnik, Proceedings of the 2nd International Conference on Quenching and the Control of Distortion, Cleveland, Ohio, November 04-07 1996, p. 181. 